\title{
A COMPUTER-ASSISTED PROOF OF THE EXISTENCE OF SMALE HORSESHOE FOR THE FOLDED-TOWEL MAP
}

\author{
ANNA GIERZKIEWICZ
}

\begin{abstract}
The paper contains a rigorous proof of existence of symbolic dynamics chaos in the generalized Hénon map's 4th iterate $H^{4}$, which was conjectured in the paper $A$ 3D Smale Horseshoe in a Hyperchaotic Discrete-Time System of Li and Yang, 2007. We prove also the uniform hyperbolicity of the invariant set with symbolic dynamics. The proofs are computer-assisted with the use of $\mathrm{C}++$ library $C A P D$ for interval arithmetic, differentiation and integration.
\end{abstract}

\section{INTRODUCTION}

The Hénon map [8] is a well known example of chaotic diffeomorphism on $\mathbb{R}^{2}$, and has been widely developed and generalized to many different contexts. Rössler in 1979 [14] introduced a similar $\mathbb{R}^{3}$ map to study hyper-chaos, which he described as 'a higher form of chaos with two directions of hyperbolic instability on the attractor'. The system gained the name of 'folded-towel map', thanks to its attractor's shape. Further generalization to $\mathbb{R}^{n}$ and its study via Lyapunov characteristic exponents may be found in [1]. For other studies of 3D Hénon-like maps by estimating their two maximal Lyapunov exponents see [6], also [13].

Our motivation is the article [12], where the 3D case with fixed parameters is considered:

$$
H(x, y, z)=\left(1.76-y^{2}-0.1 z, x, y\right) .
$$

The system (1) is also investigated, implemented as an electronic circuit, in [7]. Its 'folded towel' attractor is depicted on Fig. 1. The authors of [12] observe that the hyper-chaos may be also studied as containing a 3D generalization of Smale horseshoe dynamics on a compact subset of $\mathbb{R}^{3}$. They show explicitly two cuboids $a, b$, which most probably contain a Smale horseshoe with two expanding directions for the fourth iterate of the map (see Fig. 1, to the right).

The images of $a, b$ via $H^{4}$ have properties that can be described intuitively as follows:

- each image $H^{4}(a)$ and $H^{4}(b)$ intersects both $a$ and $b$;

- each image is compressed in the direction along the shortest edge of $a$ or $b$. This direction is 'locally normal' to the attractor;

- each image is expanded in two other directions, almost along the other edges of $a$ and $b$.

Fig. 2 shows the way the images $H^{4}(a)$ and $H^{4}(b)$ intersect $a$ and $b$.

This is a well known method for establishing (hyper-)chaos by computer assisted proof, which can be implemented with interval arithmetic (see, for example, [15, 2]). Our paper presents such a proof of the fact that the set $a \cup b$ is indeed a topological horseshoe, which implies the existence of symbolic dynamics for the system (1) (Theorem 4). Additionally we prove that the invariant set contained in $a \cup b$ is uniformly hyperbolic (Theorem 6).

The proofs of Theorems 4, 6 are computer-assisted. It means that their essential parts are $\mathrm{C}++$ codes available on-line [4]. We are aware that such codes get outdated quickly 

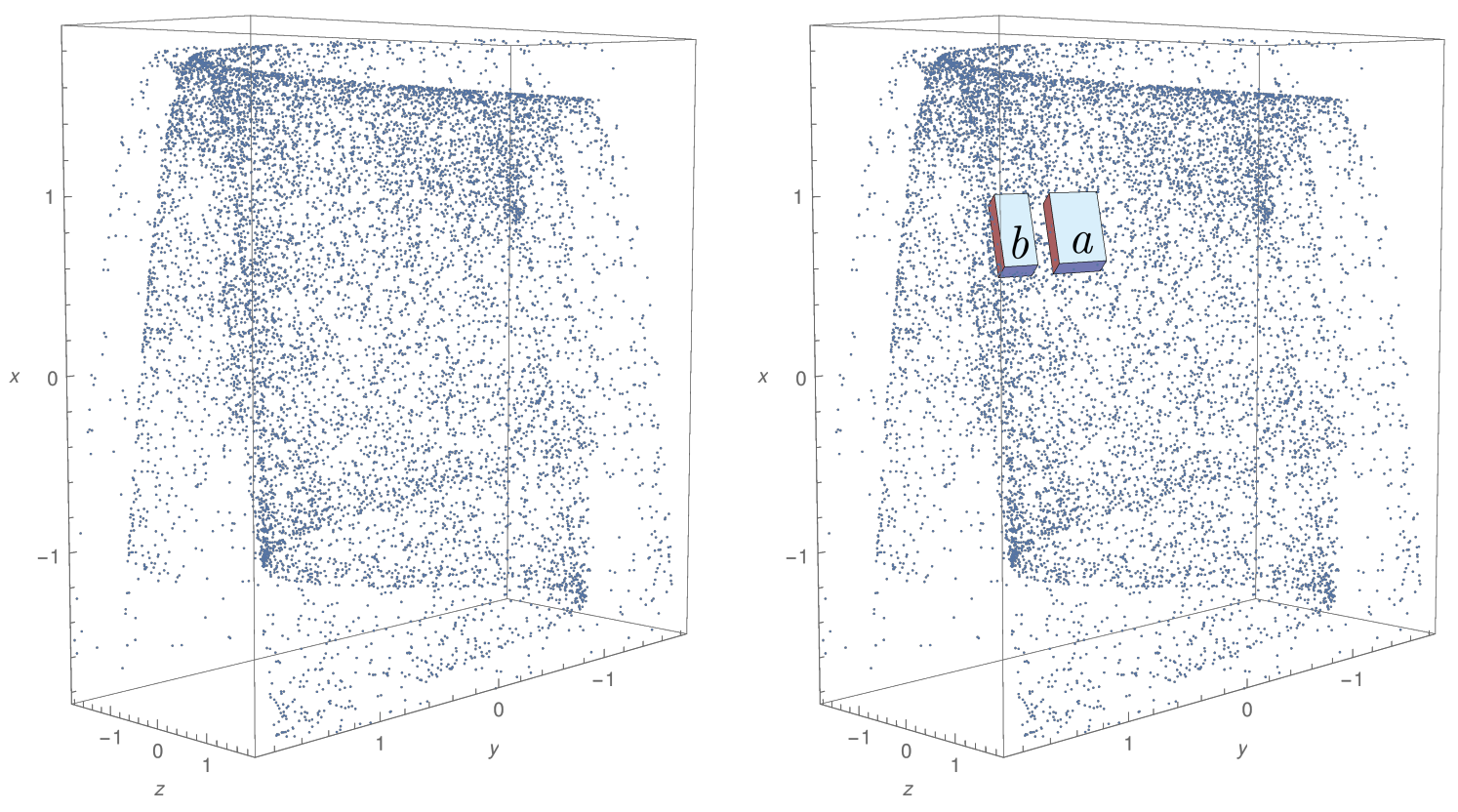

Figure 1. The 'folded towel' attractor for Hénon 3D map $H$ defined by Eq. (1).

To the right: The location of the sets $a, b$ on the attractor.
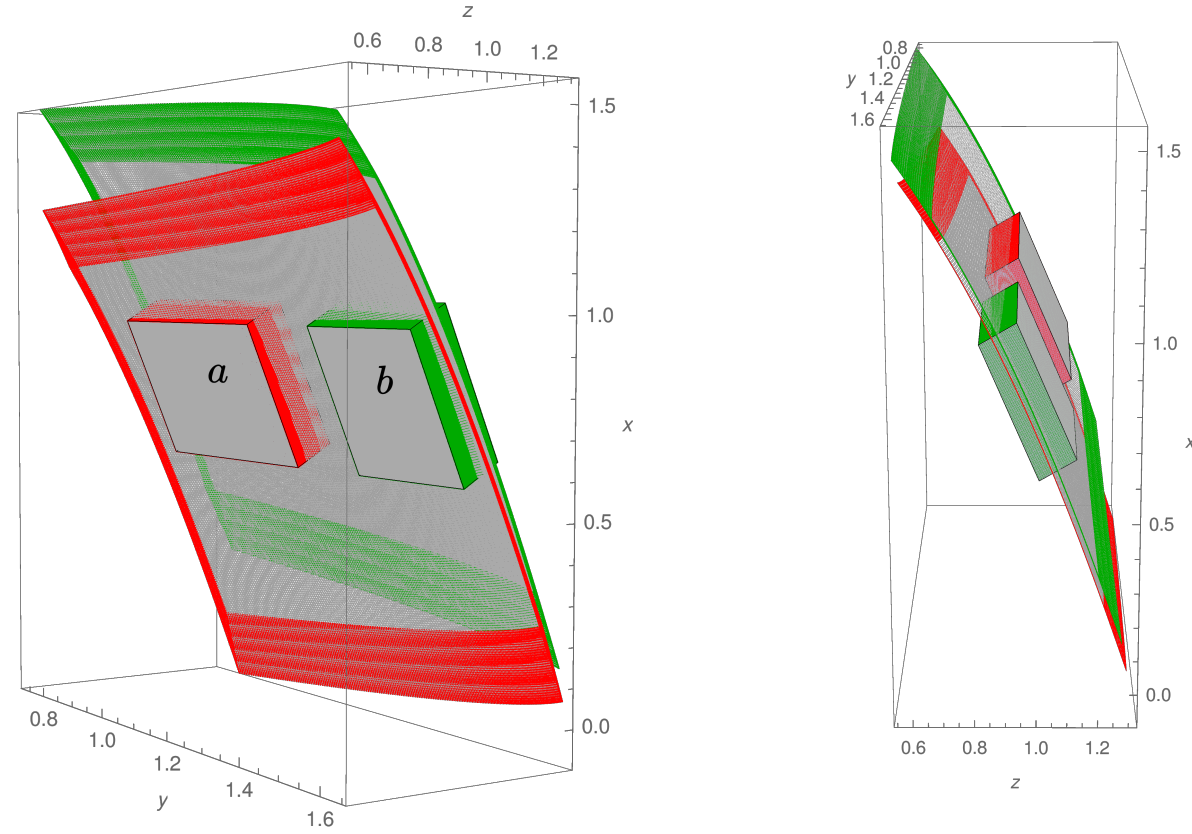

Figure 2. The location of the images $H^{4}(a), H^{4}(b)$ with respect to sets $a$ and $b$ - three-quarter view to the left and side view to the right. The exit sets and their images are marked in red (for $a$ ) and green (for $b$ ).

and after a few years they may not compile with the current $\mathrm{C}++$ compilers any more. We hope, however, that outlines of the proofs will help to understand the idea and the general construction of the codes. 


\section{TOPOLOGICAL COVERING AND PERIODIC ORBITS}

For full description of h-sets and their covering relations see [17. In [5] one may also find a 2D simplified version, which may be useful for understanding the general idea. Here we introduce a short collection of necessary notions.

We use the standard notation for the closure, interior, and boundary of a topological set $A \subset \mathbb{R}^{k}$, which are $\bar{A}$, int $A$, and $\partial A$, respectively. The $k$-dimensional open unit ball centred at the origin is denoted by $\mathbb{B}_{k}$. We use the balls in the maximum norm (cubes), for they are easily interpreted in the interval algebra as interval vectors:

$$
\mathbb{B}_{k}=\left\{\mathbf{x} \in \mathbb{R}^{k}:\|\mathbf{x}\|_{\infty}<1\right\} .
$$

2.1. H-sets. The basic object we work on is

Definition 1 ([17], Def. 3.1). An h-set is a quadruple $N=\left(|N|, u(N), s(N), C_{N}\right)$, where $|N|$ is a compact subset of $\mathbb{R}^{n}$, which we will call the support of an h-set and

(1) two numbers $u(N), s(N) \in \mathbb{N} \cup\{0\}$ complement the dimension of space:

$$
u(N)+s(N)=n ;
$$

we will call them the exit and entry dimension, or unstable and stable dimension, respectively;

(2) the homeomorphism $C_{N}: \mathbb{R}^{n} \rightarrow \mathbb{R}^{n}=\mathbb{R}^{u(N)} \times \mathbb{R}^{s(N)}$ is such that

$$
C_{N}(|N|)=\overline{\mathbb{B}_{u(N)}} \times \overline{\mathbb{B}_{s(N)}} .
$$

The support of an h-set is sometimes called simply an h-set, if it does not lead to confusion or its structure is unimportant. We also often use the notation ' $f(N)$ ' interchangeably with ' $f(|N|)$ ' to simplify formulas.

Let us set also some useful notations:

$$
\begin{aligned}
\operatorname{dim} N & =n, \\
N_{c} & =\overline{\mathbb{B}_{u(N)}} \times \overline{\mathbb{B}_{s(N)}}, \\
N_{c}^{-} & =\partial \mathbb{B}_{u(N)} \times \overline{\mathbb{B}_{s(N)}}, \\
N_{c}^{+} & =\overline{\mathbb{B}_{u(N)}} \times \partial \mathbb{B}_{s(N)}, \\
N^{-} & =C_{N}^{-1}\left(N_{c}^{-}\right), \quad N^{+}=C_{N}^{-1}\left(N_{c}^{+}\right) .
\end{aligned}
$$

Therefore, we can assume that an h-set is a product of two unitary balls moved to some coordinate system with the exit set $N^{-}$and entrance set $N^{+}$distinguished. The notions with the subscript ${ }_{c}$ refer to the 'straight' coordinate system in the image of $C_{N}$.

2.2. Covering relation. We define the topological covering:

Definition 2 ([17], Def. 3.4, simplified). Let two h-sets $M, N$ be such that $u(M)=$ $u(N)=u$ and $s(M)=s(N)=s$. For a continuous map $f:|M| \rightarrow \mathbb{R}^{n}$ denote $f_{c}=$ $C_{N} \circ f \circ C_{M}^{-1}: M_{c} \rightarrow \mathbb{R}^{u} \times \mathbb{R}^{s}$.

We say that $M f$-covers the h-set $N$ (denoted shortly as $M \stackrel{f}{\Longrightarrow} N$ ) if there exists a continuous homotopy $h:[0,1] \times M_{c} \rightarrow \mathbb{R}^{u} \times \mathbb{R}^{s}$, such that:

(1) $h_{0}=f_{c}$

(2) $h\left([0,1], M_{c}^{-}\right) \cap N_{c}=\varnothing \quad$ (the exit condition),

(3) $h\left([0,1], M_{c}\right) \cap N_{c}^{+}=\varnothing \quad$ (the entry condition). 
(4) Additionally, if $u>0$, then there exists a linear map $A: \mathbb{R}^{u} \rightarrow \mathbb{R}^{u}$ such that

$$
\begin{aligned}
& h_{1}(x, y)=(A(x), 0) \quad \text { for } x \in \overline{\mathbb{B}_{u}} \text { and } y \in \overline{\mathbb{B}_{s}}, \\
& A\left(\partial \mathbb{B}_{u}\right) \subset \mathbb{R}^{u} \backslash \mathbb{B}_{u} .
\end{aligned}
$$

For some geometrical intuition of covering in low-dimensional cases, see Figs. 3, 4 .
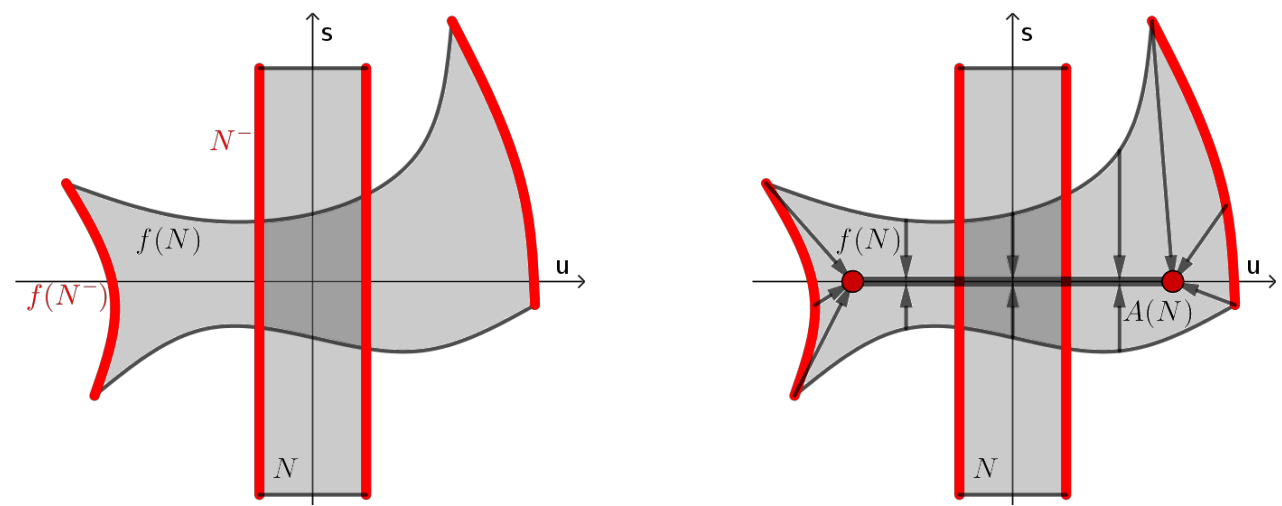

Figure 3. To the left: an example of topological self-covering $N \stackrel{f}{\Longrightarrow} N$ in $\mathbb{R}^{2}$. To the right: $f(N)$ is homotopy equivalent to the image of $N$ via a linear map $A$ with certain properties (see Definition 2). The exit sets and their images are marked in red.
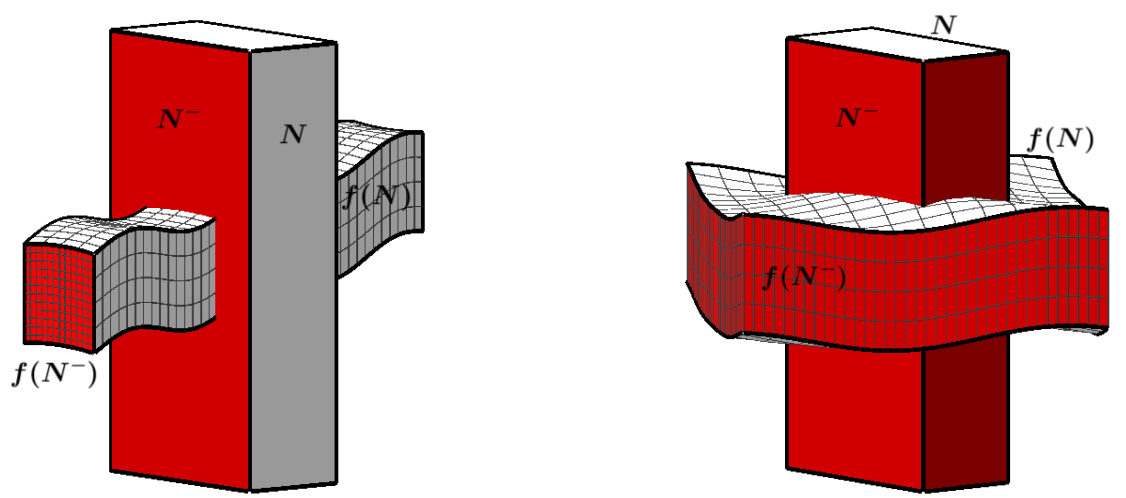

FIGURE 4. Examples of topological self-covering $N \stackrel{f}{\Longrightarrow} N$ in $\mathbb{R}^{3}$ : with one exit direction (left) and two exit directions (right). The exit sets and their images are marked in red.

Topological covering has a property of tracking orbits [10. In other words, for a chain covering $N_{1} \stackrel{f}{\Longrightarrow} N_{2} \stackrel{f}{\Longrightarrow} N_{3}$, one can find a point in $N_{1}$ that is mapped into $N_{2}$ and then to $N_{3}$. Moreover, one can prove the existence of a periodic orbit related to a closed sequence of covering relations:

Theorem 1 ([17], Theorem 3.6, simplified). Suppose there exists a sequence of h-sets $N_{0}$, $\ldots, N_{n}=N_{0}$, such that

$$
N_{0} \stackrel{f}{\Longrightarrow} N_{1} \stackrel{f}{\Longrightarrow} \ldots \stackrel{f}{\Longrightarrow} N_{n}=N_{0},
$$

then there exists a point $x \in \operatorname{int}\left|N_{0}\right|$, such that $f^{k}(x) \in \operatorname{int}\left|N_{k}\right|$ for $k=0,1, \ldots, n$ and $f^{n}(x)=x$. 
In particular, if $N_{0} \stackrel{f}{\Longrightarrow} N_{0}$, then there exists a stationary point for the map $f$, contained in $N_{0}$.

\section{Detecting Symbolic Dynamics Via COVERING Relations}

We shall prove that the map (1) is chaotic in the sense of symbolic dynamics for $H^{4}$. First let us recall this notion:

3.1. Symbolic dynamics. Let $\Sigma_{2}=\{0,1\}^{\mathbb{Z}}$ be the set of bi-infinite sequences of two symbols understood as a compact space with the metric

$$
\text { for } l=\left\{l_{n}\right\}_{n \in \mathbb{Z}}, \quad l^{\prime}=\left\{l_{n}^{\prime}\right\}_{n \in \mathbb{Z}}, \quad \operatorname{dist}\left(l, l^{\prime}\right)=\sum_{n=-\infty}^{+\infty} \frac{\left|l_{n}-l_{n}^{\prime}\right|}{2^{|n|}},
$$

which induces the product topology. The homeomorphism $\sigma: \Sigma_{2} \rightarrow \Sigma_{2}$, given by

$$
(\sigma(l))_{n}=l_{n+1},
$$

is called the shift map. It has many interesting topological properties, in particular: the existence of dense orbits, or the density of periodic orbits' set in the whole space.

In our study, by the chaotic behaviour of a discrete dynamical system induced by a homeomorphism $f: X \rightarrow X$ we understand the existence of a compact set $I \subset X$ invariant for $f$ (or sometimes its higher iterate) such that $\left.f\right|_{I}$ is semi-conjugate to $\sigma$, i.e. there exists a continuous surjection $g: I \rightarrow \Sigma_{2}$ such that

$$
\left.g \circ f\right|_{I}=\sigma \circ g \text {. }
$$

In other words, $f$ admits on $I$ at least as rich dynamics as $\sigma$ on $\Sigma_{2}$. It means, in particular, that the topological entropy of $f$ is greater or equal $\log 2$. The system $\left(\Sigma_{2}, \sigma\right)$ or any system semi-conjugate to it is often called in literature a symbolic dynamics system [11.

Symbolic dynamics is sometimes used as one of the definitions of chaotic dynamics, because the discrete system $\left(\Sigma_{2}, \sigma\right)$ defined above evinces all the typical chaotic phenomena as transitivity, density of periodic orbits set or sensitivity to initial conditions. Also, there exists a periodic orbit of any prescribed period [11].

3.2. Topological horseshoe. In general, to isolate a set with symbolic dynamics does not seem to be an easy task. It occurs, however, that some sets which fulfil certain covering relations must contain symbolic dynamics. An important example is:

Definition 3 (Topological horseshoe). Let $N_{0}, N_{1} \subset \mathbb{R}^{n}$ be two disjoint h-sets. We say that a continuous map $f: \mathbb{R}^{n} \rightarrow \mathbb{R}^{n}$ is a topological horseshoe for $N_{0}, N_{1}$ if (see Fig. 5)

$$
\begin{aligned}
& N_{0} \stackrel{f}{\Longrightarrow} N_{0}, \quad N_{0} \stackrel{f}{\Longrightarrow} N_{1}, \\
& N_{1} \stackrel{f}{\Longrightarrow} N_{0}, \quad N_{1} \stackrel{\stackrel{f}{\Longrightarrow}}{=} N_{1} .
\end{aligned}
$$

It can be shown that for any topological horseshoe we obtain symbolic dynamics.

Theorem 2 ([19], Theorem 18). Let $f$ be a topological horseshoe for $N_{0}, N_{1}$. Denote by $I=\operatorname{Inv}\left(N_{0} \cup N_{1}\right)$ the invariant part of the set $N_{0} \cup N_{1}$ under $f$, and define a map $g: I \rightarrow \Sigma_{2}$ by

$$
g(x)_{k}=j \in\{0,1\} \quad \text { iff } \quad f^{k}(x) \in N_{j} .
$$

Then $g$ is a surjection satisfying $\left.g \circ f\right|_{I}=\sigma \circ g$ and therefore $f$ is semi-conjugate to the shift map $\sigma$ on $\Sigma_{2}$. 

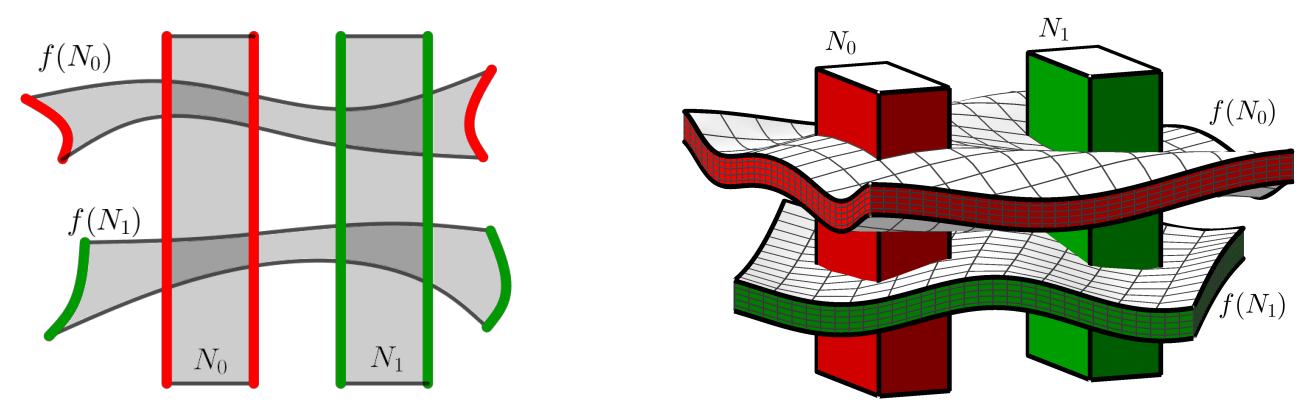

Figure 5. Topological horseshoes in $\mathbb{R}^{2}$ and $\mathbb{R}^{3}$ : each $N_{0,1}$ covers itself and the other set. The exit sets of $N_{0}$ and $N_{1}$ are marked in red and green, respectively. Compare also to Fig. 2 .

The conjugacy to the model space $\Sigma_{2}$ may be understood as follows: for any sequence of the symbols 0 and 1 there exists an orbit of the discrete system generated by $f$ passing through the sets $N_{0}$ and $N_{1}$ in the order given by the sequence. Moreover, if the sequence is $k$-periodic, then so is the orbit.

Corollary 3. Let $f$ be a topological horseshoe for $N_{0}, N_{1}$. Then it follows from Theorem 1 that for any finite sequence of zeros and ones $\left(l_{0}, l_{1}, \ldots, l_{n-1}\right), l_{i} \in\{0,1\}$, there exists $x \in N_{l_{0}}$ such that

$$
f^{i}(x) \in \operatorname{int} N_{l_{i}} \quad \text { and } \quad f^{n}(x)=x .
$$

3.3. The theorem on symbolic dynamics for Hénon 3D map. In our case, we assume that h-sets $N_{i}$ are contained in $\mathbb{R}^{3}$ and the continuous map $f=H^{4}: \mathbb{R}^{3} \rightarrow \mathbb{R}^{3}$ is such that $N_{i}$ have exit dimensions equal to 2 , that is $u\left(N_{i}\right)=u=2$ and $s\left(N_{i}\right)=s=1$ (as on the right part of Fig. 4).

The sets $a, b$ defined in [12] are parallelepipeds spanned by the sets of vertices (Fig. 1)

- $a: \quad\{(0.84,1.13,0.65),(1.205,1.13,0.65),(1.205,0.94,1.03),(0.84,0.94,1.03)$,

$(0.84,1.01,0.59),(1.205,1.01,0.59),(1.205,0.82,0.97),(0.84,0.82,0.97)\}$;

- $b: \quad\{(1.365,1.13,0.65),(1.61,1.13,0.65),(1.61,0.94,1.03),(1.365,0.94,1.03)$,

$(1.365,1.01,0.59),(1.61,1.01,0.59),(1.61,0.82,0.97),(1.365,0.82,0.97)\}$.

Define two h-sets $N_{a}, N_{b} \subset \mathbb{R}^{3}$ with supports $a$ and $b$, respectively. The supports are images of the cube $B=\left(N_{a}\right)_{c}=\left(N_{b}\right)_{c}=\overline{\mathbb{B}_{2}} \times \overline{\mathbb{B}_{1}}=[-1,1]^{3}$ by the following affine transformations:

$$
\begin{aligned}
& \left|N_{a}\right|=a=C_{a}^{-1}(B)=\left[\begin{array}{c}
0.81 \\
1.0225 \\
0.975
\end{array}\right]+\left[\begin{array}{ccc}
0 . & 0.19 & -0.03 \\
0.1825 & 0 . & 0 . \\
0 . & -0.095 & -0.06
\end{array}\right] \cdot B, \\
& \left|N_{b}\right|=b=C_{b}^{-1}(B)=\left[\begin{array}{c}
0.81 \\
1.4875 \\
0.975
\end{array}\right]+\left[\begin{array}{ccc}
0 . & 0.19 & -0.03 \\
0.1225 & 0 . & 0 . \\
0 . & -0.095 & -0.06
\end{array}\right] \cdot B,
\end{aligned}
$$

Theorem 4. For h-sets $N_{a}, N_{b}$ defined above the following chain of covering relations occurs:

$$
N_{a} \stackrel{H^{4}}{\Longrightarrow} N_{a} \stackrel{H^{4}}{\Longrightarrow} N_{b} \stackrel{H^{4}}{\Longrightarrow} N_{b} \stackrel{H^{4}}{\Longrightarrow} N_{a}
$$

which proves the existence of symbolic dynamics for the fourth iterate of map $H$, defined by (1). 
Proof. Using the CAPD library for $\mathrm{C}++[3]$ one is able to calculate the (over-estimated) image of the h-set $N$ through the map $f$ and enclose it in a cuboid (an interval closure of $f(N)$, denoted by $[f(N)])$.

To prove each of four covering relations $N_{0} \stackrel{f}{\Longrightarrow} N_{1}$, where $f=H^{4}, N_{0}, N_{1} \in\left\{N_{a}, N_{b}\right\}$ (as in (5)), we check a sufficient condition, which is a conjunction of two:

(I) $\left[f\left(N_{0}\right)\right]$ is spanned across $N_{1}$, that is:

- the image projected on any unstable coordinate (1 or 2 ) lies outside $N_{1}$ :

$$
\left\|\left[C_{N_{1}} \circ f \circ C_{N_{0}}^{-1}(B)\right]_{1,2}\right\|>1 ;
$$

- or else, the image projected on stable coordinate (3) lies between the two components of $N_{1}^{+}$:

$$
\left\|\left[C_{N_{1}} \circ f \circ C_{N_{0}}^{-1}(B)\right]_{3}\right\|<1 .
$$

(II) The estimated image of the exit set $\left[f\left(N_{0}^{-}\right)\right]$lies outside $N_{1}$ and it is homotopy equivalent to the estimated image of $N_{0}^{-}$through a chosen linear map $(A, 0)$.

The linear map $A$ satisfying the conditions from Def. 2 that we choose is $A=$ $D\left(C_{N_{1}} \circ f \circ C_{N_{0}}^{-1}\right)(0)_{u}$. Fig. 6 compares, as an example, the images of the box $B$ via maps $C_{a} \circ H^{4} \circ C_{a}^{-1}$ and $\left(A=D\left(C_{a} \circ H^{4} \circ C_{a}^{-1}\right)(0)_{u}, 0\right)$.

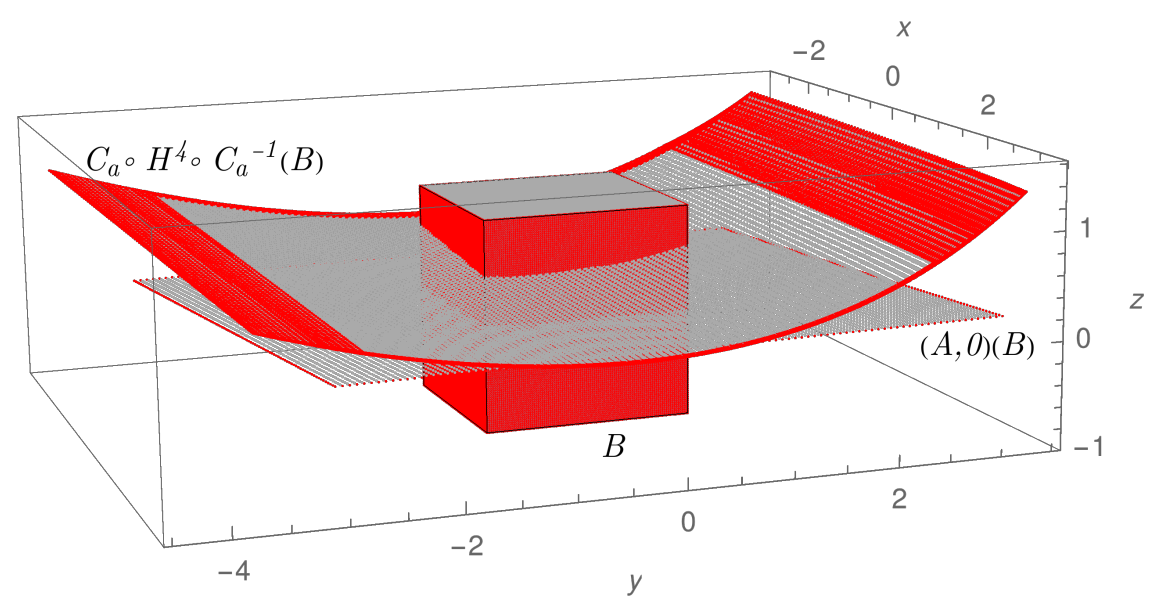

Figure 6 . The images of the box $B$ via maps $C_{a} \circ H^{4} \circ C_{a}^{-1}$ and $(A=$ $\left.D\left(C_{a} \circ H^{4} \circ C_{a}^{-1}\right)(0)_{u}, 0\right)$. The exit set and its images are marked in red.

Next, we fix one of the unstable dimensions (1 and subsequently 2$)$ and check for each of two faces of $\left(N_{0}^{-}\right)_{1,2}$, if the interval hull of the union of its image through $f$ and through $(A, 0)$ lies outside $N_{1}$, projected on the considered coordinate:

$$
\left\|\left[\left[C_{N_{1}} \circ f \circ C_{N_{0}}^{-1}\left(B_{(\text {face })}^{-}\right)\right] \cup\left[C_{N_{1}} \circ(A, 0) \circ C_{N_{0}}^{-1}\left(B_{(\text {face })}^{-}\right)\right]\right]_{1,2}\right\|>1
$$

This means, in particular, that there exists a homotopy

$$
h:[0,1] \times \overline{\mathbb{B}_{2}} \times \overline{\mathbb{B}_{1}} \ni(t, x, y) \longmapsto(1-t) f(x, y)+t(A(x), 0) \in \mathbb{R}^{3}
$$

connecting the images of the face through $f$ and through $(A, 0)$ which does not touch $N_{1}$. 
Outline of the computer-assisted proof [4].

The program 03a_Henon_Towel_Symbolic_Dynamics.cpp consists of the following steps:

(1) We define the interval map $H^{4}$ and h-sets $N_{a}, N_{b}$.

(2) We check each of four covering relations $N_{0} \stackrel{f}{\Longrightarrow} N_{1}$, where $f=H^{4}, N_{0}, N_{1} \in$ $\left\{N_{a}, N_{b}\right\}$ by:

(a) dividing $N_{0}$ in $20 \times 20 \times 20$ small h-sets and checking the condition (I) for each part,

(b) dividing each of two faces of $\left(N_{0}^{-}\right)_{1}$ in $10 \times 10$ small parts and checking the condition (II) for every part in unstable dimension 1 ,

(c) dividing each of two faces of $\left(N_{0}^{-}\right)_{2}$ in $10 \times 10$ small parts and checking the condition (II) for every part in unstable dimension 2 .

(3) The above conditions are fulfilled, proving every relation $N_{0} \stackrel{H^{4}}{\Longrightarrow} N_{1}$, where $N_{0}, N_{1} \in\left\{N_{a}, N_{b}\right\}$. Therefore, Theorem 4 is proved.

\section{HYPERBOLICITY}

4.1. Uniform and strong hyperbolicity. First, recall the notion of uniform hyperbolicity. Let $f: \mathbb{R}^{n} \rightarrow \mathbb{R}^{n}$ be a diffeomorphism and $I \subset \mathbb{R}^{n}$ - a compact invariant set for $f$.

Definition 4 (9]). We say that $f$ is uniformly hyperbolic on $I$ if for every point $x \in I$ the tangent space $T_{x} I$ is equal to a direct sum $T_{x} I=E_{x}^{u} \oplus E_{x}^{s}$ such that

$$
D f(x) E_{x}^{u}=E_{f(x)}^{u}, \quad D f(x) E_{x}^{s}=E_{f(x)}^{s},
$$

and for some constants $c>0,0<\lambda<1$ independent of $x$ the inequalities

$$
\begin{aligned}
& \forall v \in E_{x}^{s} \quad\left\|D f^{k}(x) v\right\|<c \lambda^{k}\|v\|, \\
& \forall v \in E_{x}^{u} \quad\left\|D f^{-k}(x) v\right\|<c \lambda^{k}\|v\|
\end{aligned}
$$

hold for every $k \geq 0$.

In [18 and [16] the authors introduce a method for proving hyperbolicity using the notion of $h$-set with cones. In our case, we shall not need this definition except some auxiliary notations.

Denote by $Q$ the $n \times n=(u+s) \times(u+s)$ block matrix

$$
Q=\left[\begin{array}{cc}
\operatorname{Id}_{u} & 0 \\
0 & -\mathrm{Id}_{s}
\end{array}\right]
$$

where by $\operatorname{Id}_{k}$ we mean the identity matrix of dimension $k \times k$.

Suppose now that we have a set $M=\bigcup_{i=1}^{N} M_{i}$, where $M_{i} \subset \mathbb{R}^{n}$ are compact and have pairwise disjoint interiors. Each $M_{i}$ is related to an affine coordinate system $C_{i}$. Let $I$ be the invariant part of $M$ for the diffeomorphism $f$, that is

$$
I=\operatorname{Inv}_{f}(M)=\left\{x \in M \mid \forall_{n \in \mathbb{N}} f^{n}(x) \in M \wedge f^{-n}(x) \in M\right\} .
$$

Denote also $f_{i j}=C_{j} \circ f \circ C_{i}^{-1}$ for $i, j=1, \ldots, N$.

Definition 5 ([16], Def. 2.2). We say that $f$ is strongly hyperbolic on $M$ if for $x \in M_{i}$ and $j=1, \ldots, N$ such that $f\left(M_{i}\right) \cap M_{j} \neq \varnothing$

$$
\text { the matrix } D f_{i j}(x)^{T} \cdot Q \cdot D f_{i j}(x)-Q \quad \text { is positive definite. }
$$

Theorem 5 ([16], Th. 2.3). If $f$ is strongly hyperbolic on $M=\bigcup_{i=1}^{N} M_{i}$, then $f$ is uniformly hyperbolic on $I=\operatorname{Inv}_{f}(M)$. 


\subsection{The theorem on hyperbolicity.}

Theorem 6. The map $H^{4}$ is uniformly hyperbolic on $\operatorname{Inv}_{H^{4}}(a \cup b)$.

Proof. From the computer-assisted proof [4] we deduce that $H^{4}$ is strongly hyperbolic on $a \cup b$ and therefore the thesis follows from Theorem 5 .

Outline of the computer-assisted proof [4].

The program 04_Henon_Towel_Hyperbolicity.cpp consists of the following steps:

(1) We define four interval maps $f_{a a}=C_{a} \circ H^{4} \circ C_{a}^{-1}$, and analogously $f_{a b}, f_{b a}, f_{b b}$. We will check the condition (6) for $x \in B=C_{a}(a)=C_{b}(b)$.

(2) In our case $u=2, s=1$, so we also define

$$
Q=\left[\begin{array}{ccc}
1 & 0 & 0 \\
0 & 1 & 0 \\
0 & 0 & -1
\end{array}\right] .
$$

(3) We calculate the interval upper approximation of the matrices $D f(B)^{T} \cdot Q \cdot D f(B)-$ $Q, f \in\left\{f_{a a}, f_{a b}, f_{b a}, f_{b b}\right\}$, and check with Sylvester's criterion if they are positive definite (the conditions are not fulfilled on the whole box $B$, for the images are too much over-estimated).

(4) We divide the box $B$ into $(25 \times 25 \times 25)$ smaller boxes, and for each part $B_{i}$ and each map $f \in\left\{f_{a a}, f_{a b}, f_{b a}, f_{b b}\right\}$ we check if:

- the estimated image $\left[f\left(B_{i}\right)\right]$ has an empty intersection with $B$ : if so, then $B_{i}$ does not intersect $I$ and we do not need to check the condition (6);

- else, if the interval matrix $\left[D f\left(B_{i}\right)\right]^{T} \cdot Q \cdot\left[D f\left(B_{i}\right)\right]-Q$ is positive definite.

(5) The above conditions are fulfilled for each map $f \in\left\{f_{a a}, f_{a b}, f_{b a}, f_{b b}\right\}$ and every point in $B$. Therefore, Theorem 6 is proved.

\section{Conclusion}

We proved the existence of symbolic dynamics in $a \cup b$ for the $\mathbb{R}^{3}$ map $H^{4}$ defined by (1), and also the hyperbolicity of its invariant part $\operatorname{Inv}_{H^{4}}(a \cup b)$. We believe that the methods used in this paper can be easily applied also in other dimensions, especially for maps with two or more expanding directions.

\section{ACKNOWLEDGMENT}

The author would like to thank Professors Piotr Zgliczyński and Daniel Wilczak for all their devoted time and insightful comments during common discussions.

\section{REFERENCES}

[1] G. Baier and M. Klein. Maximum hyperchaos in generalized Hénon maps. Physics Letters A, 151(6):281 - 284, 1990 .

[2] R. Barrio, M. Angeles Martínez, S. Serrano, and D. Wilczak. When chaos meets hyperchaos: 4D Rössler model. Physics Letters A, 379(38):2300 - 2305, 2015.

[3] CAPD group. Computer Assisted Proofs in Dynamics C++ library.http://capd.ii.uj.edu.pl.

[4] A. Gierzkiewicz. C ++ source code. Available online on http://kzm.ur.krakow.pl/ agierzkiewicz/publikacje.html.

[5] A. Gierzkiewicz and P. Zgliczyński. A computer-assisted proof of symbolic dynamics in Hyperion's rotation. Celestial Mechanics and Dynamical Astronomy, 131(7), 2019.

[6] S.V. Gonchenko, I. Ovsyannikov, C. Simó, and D. Turaev. Three-dimensional Hénon-like maps and wild Lorenz-like attractors. International Journal of Bifurcation and Chaos, 15, 112011. 
[7] G. Grassi and D.A. Miller. Theory and experimental realization of observer-based discrete-time hyperchaos synchronization. IEEE Transactions on Circuits and Systems I: Fundamental Theory and Applications, 49(3):373-378, 2002.

[8] M. Hénon. A two-dimensional mapping with a strange attractor. Comm. Math. Phys., 50(1):69-77, 1976.

[9] M. Hirsch, C. Pugh, and M. Shub. Invariant Manifolds, volume 76. 011977.

[10] C. Miranda. Un'osservazione su un teorema di Brouwer. Pubblicazioni (Istituto per le Applicazioni del Calcolo). Consiglio Nazionale delle Ricerche, 1940.

[11] M. Morse and G. Hedlund. Symbolic dynamics. Amer. J. Math., 60:815-866, 1938.

[12] L. Qingdu and Y. Xiao-Song. A 3D Smale horseshoe in a hyperchaotic discrete-time system. Discrete Dynamics in Nature and Society, 2007, 012007.

[13] H. Richter. The generalized Hénon maps: Examples for higher-dimensional chaos. I. J. Bifurcation and Chaos, 12:1371-1384, 062002.

[14] O.E. Rössler. An equation for hyperchaos. Physics Letters A, 71(2):155 - 157, 1979.

[15] D. Wilczak. Abundance of heteroclinic and homoclinic orbits for the hyperchaotic Rössler system. Discrete and Continuous Dynamical Systems - series B, 11, 062009.

[16] D. Wilczak. Uniformly hyperbolic attractor of the Smale-Williams type for a Poincaré map in the Kuznetsov system. SIAM J. Applied Dynamical Systems, 9:1263-1283, 2010.

[17] D. Wilczak and P. Zgliczyński. Heteroclinic connections between periodic orbits in planar restricted circular three-body problem - a computer assisted proof. Communications in Mathematical Physics, 234(1):37-75, Mar 2003.

[18] P. Zgliczyński. Covering relations, cone conditions and the stable manifold theorem. Journal of Differential Equations, 246(5):1774-1819, 2009.

[19] P. Zgliczyński and M. Gidea. Covering relations for multidimensional dynamical systems. Journal of Differential Equations, 202(1):32-58, 2004.

Email address: anna.gierzkiewicz@urk.edu.pl

Department of Applied Mathematics, University of Agriculture in Kraków, ul. BalICKA 253C, 30-198 KRAKów, POLAND 